\title{
Photonic Crystal Coupled to N-V Center in Diamond
}

\author{
Luca Marseglia \\ Additional information is available at the end of the chapter
}

http://dx.doi.org/10.5772/54138

\section{Introduction}

In this work we aim to exploit one of the most studied defect color centers in diamond , the negatively charged nitrogen vacancy $\left(\mathrm{NV}^{-}\right)$color center, a three level system which emits a single photon at a wavelength of $637 \mathrm{~nm}$ providing a possible deterministic single photon emitter very useful for quantum computing applications. Moreover the possibility of placing a $\mathrm{NV}^{-}$in a photonic crystal cavity will enhance the coupling between photons and $\mathrm{NV}^{-}$center. This could also allow us to address the ground state of the $\mathrm{NV}^{-}$center, whose spin, could be used as qubit. It is also remarkable to notice that for quantum computing purposes it is very useful to increase the light collection from the $\mathrm{NV}^{-}$centers, and in order to do that we performed a study of another structure, the solid immersion lens, which consists of an hemisphere whose center is at the position of an emitter, in this case the $\mathrm{NV}^{-}$center, increasing the collection of the light from it. In order to create these structures we used a method called focused ion beam which allowed us to etch directly into the diamond many different kinds of structures. In order to allow an interaction between these structures and the $\mathrm{NV}^{-}$centers we need to have a method to locate the $\mathrm{NV}^{-}$center precisely under the etched structures. We developed a new technique ([1]) where we show how to mark a single $\mathrm{NV}^{-}$center and how to etch a desired structure over it on demand. This technique gave very good results allowing us to etch a solid immersion lens onto a $\mathrm{NV}^{-}$previously located and characterized, increasing the light collection from the $\mathrm{NV}^{-}$of a factor of $8 \times$.

\section{Introduction to nitrogen vacancy center in diamond}

Diamond has emerged in recent years as a promising platform for quantum communication and spin qubit operations as shown by [2], as well as for "quantum imaging" based on single spin magnetic resonance or nanoscopy. Impressive demonstrations in all these areas have mostly been based on the negatively-charged nitrogen vacancy center, $\mathrm{NV}^{-}$, which consists of a substitutional nitrogen atom adjacent to a carbon vacancy. Due to its useful optical and magnetic spin selection properties, the $\mathrm{NV}^{-}$center has been used by [3] to demonstrate a stable single photon source and single spin manipulations ([4]) at room temperature. A single-photon source based on $\mathrm{NV}^{-}$in nano-diamond is already commercially available, and a ground state spin coherence time of $15 \mathrm{~ms}$ has been observed in ultra-pure diamond at 


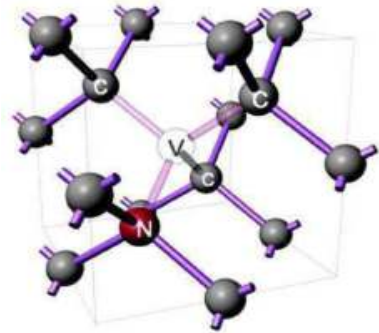

(a)
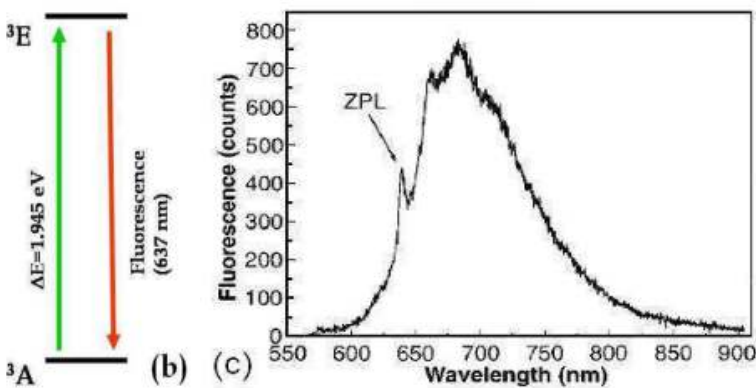

Figure 1. a)Atomic structure of $\mathrm{NV}^{-}$center in diamond( $\mathrm{N}=$ nitrogen $\left.\mathrm{V}=\mathrm{Vacancy}, \mathrm{C}=\mathrm{Carbon}\right)$ b) Energy level scheme of $\mathrm{NV}^{-}$center. c) Fluorescence spectrum of a single $\mathrm{NV}^{-}$defect center. The wavelength of the zero phonon line ( ZPL) is $637 \mathrm{~nm}$ $(1.945 \mathrm{eV})$. Excitation was at 514nm [6]

room temperature. At present, one of the biggest issues preventing diamond from taking the lead among competing technologies is the difficulty in fabricating photonic devices to couple and guide light. For the realization of large-scale quantum information processing protocols (e.g. via photonic module approaches) or for quantum repeater systems, it will be necessary to connect $\mathrm{NV}^{-}$centers through "flying" qubits such as photons. To achieve this, micro-cavities and waveguides are needed to enable the transfer of quantum information between the electron spin of the $\mathrm{NV}^{-}$center and a photon. In this work I will show some applications of diamond useful for quantum computing. Synthetic diamonds can be doped in order to create implanted $\mathrm{NV}^{-}$center which interacts with light, as described further. From its discovery, it has not been very clear if the $\mathrm{NV}^{-}$were a proper two level system. Recently it has been shown that it has properties more typical of a three level system with a metastable level. In its ground state it has spin $s=1$ and different emission rates for transitions to the ground states, so $\mathrm{NV}^{-}$center can be also exploited in order to achieve spin readout.

\section{Interaction of $\mathrm{N}-\mathrm{V}$ center with light}

The $\mathrm{NV}^{-}$center in diamond occurs naturally or is produced after radiation damage and annealing in vacuum. As described earlier is made by substitutional nitrogen atom adjacent to a vacancy in carbon lattice in the diamond as depicted in Fig.1a. The $\mathrm{NV}^{-}$center has attracted a lot of interest because it can be optically addressed as a single quantum system as discussed by [5]. The $\mathrm{NV}^{-}$center behaves as a two level system with a transition from the excited state to the ground state providing a single photon of $637 \mathrm{~nm}$, as shown in Fig.1b. This is a very useful characteristic for quantum information purposes because it can be used as single photon source. Let us remember that a characteristic of the $\mathrm{NV}^{-}$center is a zero-phonon line (ZPL), in the spectrum at room temperature, at $637 \mathrm{~nm}$ as shown in Fig.1c, the zero-phonon line constitutes the line shape of individual light absorbing and emitting molecules embedded into the crystal lattice. The state of $\mathrm{NV}^{-}$center ground state spin strongly modulates the rate of spontaneous emission from the ${ }^{3} E \leftrightarrow^{3} A$ sub-levels providing a mechanism for spin read out as discussed by [4]. We have recently shown theoretically ([7]) that spin readout with a small number of photons could be achieved by placing the $\mathrm{NV}^{-}$centre in a subwavelength scale micro-cavity with a moderate $Q$-factor $(Q \sim 3000)$. So one of our aims is to optimize the output coupling of photons from diamond color 


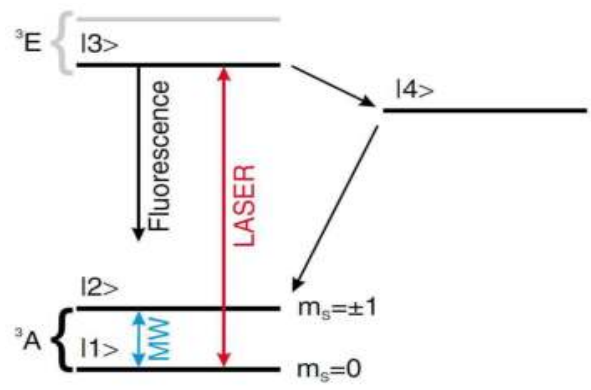

Figure 2. Energy level scheme of the nitrogen vacancy defect center in diamond. The greyed out lines correspond to the $m_{s}= \pm 1$ sublevels [10]

centers into waveguides and free space to increase the efficiency of single photon sources and to enable faster single spin read-out. In order to do that we want to study resonant structures. These structures confine the light close to the emitter allowing cavity-QED effects to be exploited to direct an emitted photon into a particular spatial mode and will allow us to enhance the ZPL. An improvement of the photon emission rate and photon indistinguishability for $\mathrm{NV}^{-}$can be achieved due to the (coherent) interaction with the highly localized photon field of the cavity. In principle a high-Q micro-cavity can be realized directly in diamond but the first experimental demonstrations with micro-disk resonators and photonic crystal cavities, made for example by [8], suffered from large scattering losses due to the poly-crystalline nature of the diamond material used. The fabrication of high-Q cavities in single crystal diamond is very challenging because vertical optical confinement within diamond requires either a $3 D$ etching process or a method for fabricating thin single crystal diamond films. We want analyze photonic crystal structures in diamond and fabrication methods to achieve efficient spin read-out in low-Q cavities. Electronic spin resonance (ESR) experiments performed by [9] has shown that the electronic ground state of $\mathrm{NV}^{-}$center $\left({ }^{3} A\right)$ is paramagnetic. Indeed the electronic ground state of the $\mathrm{NV}^{-}$center is a spin triplet that exhibits a $2.87 \mathrm{GHz}$ zero-field splitting defining the $\mathrm{z}$ axis of the electron spin. An application of a small magnetic field splits the magnetic sublevel $m_{s}= \pm 1$ energy level structure of the $\mathrm{NV}^{-}$center, as we can see in Fig.2. Electron spin relaxation times $\left(T_{1}\right)$ of defect centers in diamond range from millisecond at room temperature to seconds at low temperature. Several experiments have shown the manipulation of the ground state spin of a $\mathrm{NV}^{-}$center using optically detected magnetic resonance (ODMR) techniques, the main problem in using ODMR is that detection step involves observing fluorescence cycles from the $\mathrm{NV}^{-}$center which has a probability of destroying the spin. Another characteristic of $\mathrm{NV}^{-}$center useful for quantum information storage is the capability of transferring its electronic spin state to nuclear spins. Experiments performed by [5] have shown the possibility of manipulating nuclear spins of $\mathrm{NV}^{-}$. Nuclear spins are of fundamental importance for storage and processing of quantum information, their excellent coherence properties make them a superior qubit candidate even at room temperature.

\section{Beyond the two level system model}

In order to study the dynamics of the $\mathrm{NV}^{-}$center, remembering that $m_{e}<<m_{C}$ where $m_{e}$ is the value of the mass of the electrons and $m_{C}$ is the value of the mass of carbon atom, 


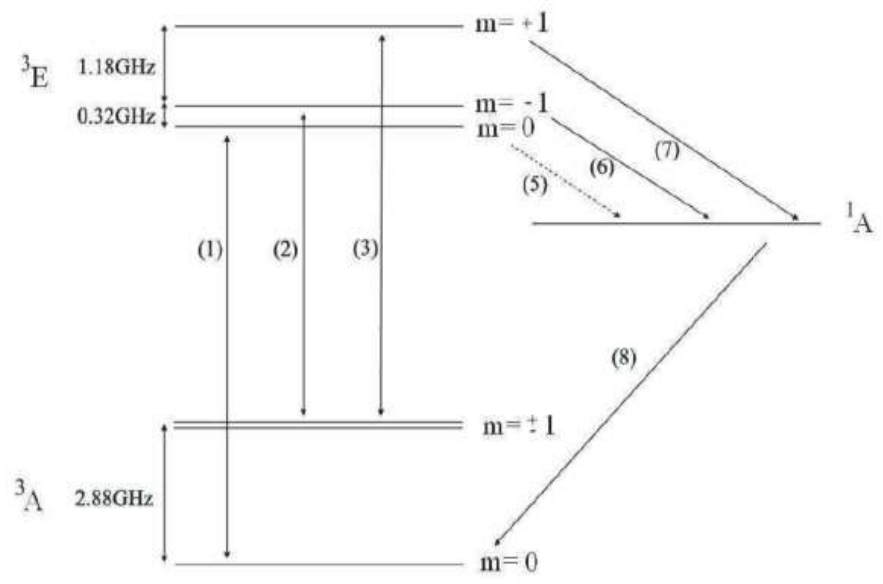

Figure 3. Experimentally measured energy level diagram of the NV center in diamond showing the experimentally determined ground and excited state splitting $[17,18]$. The defect has zero phonon line at $637 \mathrm{~nm}$, with width of order $\mathrm{MHz}$ at low temperatures [19](image taken from [7]).

we make the so called Born-Oppenheimer approximation, in which we consider the nuclei fixed in a crystal geometry and the coordinates of the electrons are considered with respect to them. When a defect is present it breaks down the crystal symmetry and, regarding the $\mathrm{NV}^{-}$center, we have a contribution of one electron from each carbon atom, the nitrogen contributes two electrons, and an extra electron comes from the environment as described by [11], possibly given by substitutional nitrogen, so ending with a total number of six electrons. The excited level of the $\mathrm{NV}^{-}$is not yet very well understood explicitly but there are many theoretical descriptions using group theory and partially confirmed by experimental results. The joint use of group theory and numerical calculations has led to predictions of the ordering of the levels of the ground state and excited states of the $\mathrm{NV}^{-}$. Taking account the coulomb interaction, spin-orbit effect and spin-spin coupling it explains the splitting of the levels initially degenerate giving rise to different transition between them. Without entering in to detailed group theory calculations we can summarize by stating that the hamiltonian of the system is composed of three elements, the coulomb interaction $H_{C}$, the spin orbit interaction $H_{S O}$ and the spin-spin interaction $H_{S S}$.

$$
H=H_{C}+H_{S O}+H_{S S}
$$

The dynamics of the system is resolved by solving the hamiltonian in free space with coulomb interaction as potential and the spin-orbit and spin-spin interaction were eventually added as perturbations. We show the detailed energy level structure of the $\mathrm{NV}^{-}$center in Fig.3. The overall effect can be summarized as follows, the coulomb interaction, splits the degeneracy between singlet and triplets of the ground state and the first excited state, the spin orbit interaction splits the states which have $M_{s=0}$ and $M_{s= \pm 1}$ and finally the spin-spin splits the A levels. The end result is that the optical transitions between ground and excited states $(1-3)$ occur at different energies. In the absence of external fields the ground state is a spin triplet split by $2.88 \mathrm{GHz}$ due to spin-spin interactions. [12] showed that the excited state is a triplet 
split by spin-spin interactions, but with the further addition of spin-orbit coupling . Recent experimental evidence performed by [13], has uncovered this excited state structure (Fig.3). The net effect of spin-spin and spin-orbit interactions is to create a detuning $\approx 1.4 \mathrm{GHz}$ $(6 \mu \mathrm{eV})$ between the transitions ${ }^{3} A_{(m=0)} \rightarrow{ }^{3} E_{(m=0)}$ (transitions 1)and ${ }^{3} A_{(m=+1)} \rightarrow{ }^{3} E_{(m=+1)}$ (transition 3). A similar detuning of $\approx 2.5 \mathrm{GHz}(10 \mu \mathrm{eV})$ exists for the ${ }^{3} A_{(m=-1)} \rightarrow{ }^{3} E_{(m=-1)}$ (transition 2), the rates for these three transition is $k_{1}=k_{2}=k_{3}=77 \mathrm{MHz}$ which gives a spontaneous emission (SE) lifetime $\tau \approx 13 n s$. The energy level structure is not simply a ground and excited triplet state, there also exists an intermediate singlet state ${ }^{1} A$ arising from Coulomb interactions. There is a probability of the transition ${ }^{3} E \rightarrow^{1} A$, with different rates depending on the spin. For the ${ }^{3} E_{m= \pm 1}$ states (transitions 6,7 ) both theoretical predictions and experimental results suggest that the decay rate is around $k_{6}=k_{7}=30 \mathrm{MHz}$ giving a spontaneous emission (SE) lifetime $\tau \approx 30 \mathrm{~ns}$. For the ${ }^{3} E_{m=0}$ state (transition 5 ) theoretically the rate of decay to the singlet should be zero, however, experimental observations made by [14] have shown the rate to be $\approx 10^{-4} \times 1 / \tau$. Since the ${ }^{1} A$ singlet state decays preferentially to the ${ }^{3} E_{m=0}$ state (transition 8), then it is clear from the rates above that broadband excitation leads to spin polarization in the spin zero ground state. Since transition 8 is non-radiative then there will be a dark period in the fluorescence when ${ }^{1} A$ becomes populated, and as the decay rate from ${ }^{3} E_{m= \pm 1}, k_{8}=3.3 \mathrm{MHz}$, to the singlet state is much larger than from ${ }^{3} E_{m=0}$, the change in intensity measures the spin state. Clearly using fluorescence intensity to detect the spin state has a probability to flip the spin, therefore it would seem necessary for a scheme to suppress this. However, spin-flip transitions are essential to initialize the system. Thus a compromise is required between the perfectly cyclic spin preserving transitions required for readout and the spin flip transitions needed for reset.

\section{Photonic crystals}

To take advantage of atom-photon coupling using $\mathrm{NV}^{-}$, as required by many quantum protocols, cavity structures are required. Again, concentrating on monolithic diamond solutions, photonic crystal cavities are the most natural structures to explore. A photonic crystal structure modulates the propagation of light in a way that is analogous to the way a semiconductor crystal modulates the motion of electrons. In both cases a periodic structure gives rise to 'band-gap' behavior, with a photon (electron) being allowed or not allowed to propagate depending on its wave vector. In photonic crystals the periodicity is comprised of regions of higher and lower dielectric constants. The basic physical phenomenon is based on diffraction, the period needs to be of the order of a half-wavelength of the light to be confined. For visible light the wavelength goes from $200 \mathrm{~nm}$ (blue) to $650 \mathrm{~nm}$ (red), leading to a real challenge in order to make the fabrication of optical photonic crystals because of the small dimensions. Breaking the periodicity in a controlled way creates nanocavities that confine light to extremely small volumes in which the lightmatter interaction is dominated by cavity quantum electrodynamic. We have previously described the characteristics of the $\mathrm{NV}^{-}$center, a three level system which is promising as an efficient room temperature source of single photons at a wavelength of $637 \mathrm{~nm}$. We pointed out that the $\mathrm{NV}^{-}$center looks very promising for performing quantum spin readout, which is also useful for quantum computing purposes. Zero-phonon emission, at $637 \mathrm{~nm}$, accounts for only a small fraction $(\sim 4 \%)$ of $\mathrm{NV}^{-}$fluorescence, with the majority of emitted photons falling in the very broad $(\sim 200 \mathrm{~nm})$ phonon-assisted sideband. By coupling the $\mathrm{NV}^{-}$center to a photonic crystal cavity, spontaneous emission in the phonon sideband can be suppressed and emission in 
the zero-phonon line can be enhanced ([15]) so the photonic crystals offers a controllable electromagnetic environment, ideal for the compact integration and isolation of the fragile quantum system. The challenges of engineering the parameters of the photonic crystal in diamond at this scale are not trivial, as described further where we will show how to tune a cavity to increase the efficiency of light collection from an emitter placed in it. Indeed, a single photon emitted by a NV${ }^{-}$could then interact with another $\mathrm{NV}^{-}$allowing entanglement between both qubits represented by the spin of the $\mathrm{NV}^{-}$centers ase described by [16]. High-Q resonators of different kinds have been fabricated in non-diamond materials and coupled to $\mathrm{NV}^{-}$emission from nano-diamonds. Since we are concerned here with developing monolithic photonics, it is necessary to fabricate cavities in the diamond itself. It should be noted that photonic crystal cavities have been fabricated in diamond films and an un-coupled Q-factor as high as 585 at $637 \mathrm{~nm}$ has been measured by [8]. The polycrystal nature of the material used in those demonstrations makes it unsuitable for our purposes due to enhanced scattering and background fluorescence. We aim to fabricate photonic crystal cavities in ultra-high-purity type IIa single-crystal diamond (Element Six) grown by chemical vapor deposition. This material has extremely low levels of nitrogen (less than $1 p p b$ ), and very few native $\mathrm{NV}^{-}$centers, making it the ideal material for creating $\mathrm{NV}^{-}$centers in a controlled fashion by implantation and annealing. In order to have strong coupling we need to have a cavity with high $\mathrm{Q}$ factor and small modal volume, but a cavity with a more moderate $\mathrm{Q}$ would still be useful. In particular, a scheme for reading out the ground state spin of an $\mathrm{NV}^{-}$center has been described by our group ([7]), that requires a $Q$ (before coupling) of only $\sim 3000$. This scheme exploits the zero-field splitting in the $\mathrm{NV}^{-}$center ground state and uses narrow band resonant excitation to achieve high-fidelity read-out of the ground state spin with just a few excitation cycles.

\section{Two-dimensional hexagonal photonic crystal structure}

Our aim is to fabricate a structure which will behave as resonant cavity for the single photon emission of the $\mathrm{NV}^{-}$center. The best choice to pursue this goal would have been a $3 D$ photonic crystal structure with a $\mathrm{NV}^{-}$placed in its center, but unfortunately the fabrication of this kind of structure is challenging. So we decide to follow a different path using a quasi-3D structure. In fact combining the photonic crystal feature and the total internal reflection $(T I R)$, we obtain a structure which confines the light in the three directions XYZ. Indeed the light is confined by distributed Bragg reflection in the plane of periodicity $(X Y)$ and by total internal reflection in the perpendicular plane $(Z)$, so we aim to fabricate a photonic crystal in a thin membrane. Recently [17]we have described in detail the study of a photonic cristal cavity, here we report just the results obtained for the $L 3$ structure which consist of a mebrane of $185 \mathrm{~nm}$ with two-dimensional hexagonal photonic crystal structure which has a bandgap centered to wavelength of $637 \mathrm{~nm}$, namely a PC structure resonant with the $\mathrm{NV}^{-}$center emission, as shown in is shown in Fig.4a with its calculated resonance frequency shown in Fig.4b.

\section{Focussed ion beam milling}

The system we used to etch structures into the diamond is a well-studied and developed technology known as focussed ion beam milling $(F I B)[18]$. It was initially developed during the late 1970s and the early 1980s, and the first commercial instruments were introduced 

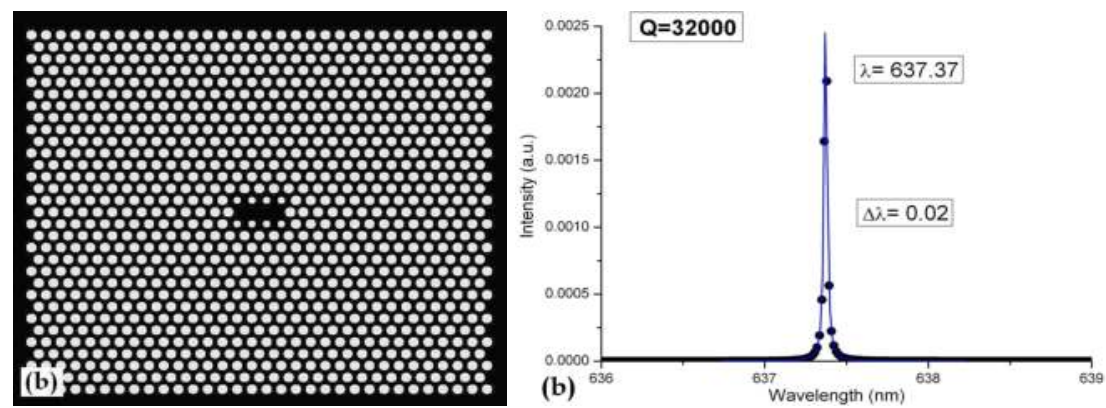

Figure 4. a) $L 3$ photonic crystal cavity structure modified with smaller radius and nearest holes shifted. b) Fourier Transform of the $E_{x}$ plot with Lorentzian Fit leading to an estimation of $Q=32000$

more than a decade ago [19]. The technology enables localised milling and deposition of conductors and insulators. A schematic diagram of a FIB column is shown in Fig. 5. The structure of the column is similar to that of a scanning electron microscope, with the difference that the $F I B$ machine uses $\mathrm{Ga}^{+}$ions instead of electrons. Inside the column a vacuum of about $1 \times 10^{-7} \mathrm{mbar}$ is maintained. In the $F I B$ system a highly focussed ion beam (diameter $\sim 10 \mathrm{~nm}$ ) is aimed at a target area on the sample, the ion beam is generated from a liquid-metal ion source (LMIS) by the application of a strong electric field.

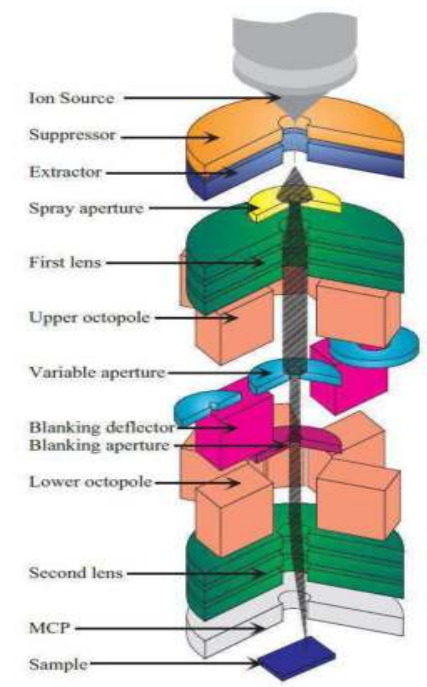

Figure 5. : Schematic diagram of a FIB column (image taken from [20]).

This electric field causes the emission of positively charged ions from a liquid gallium cone, which is formed on the tip of a tungsten needle. After being focused with the first collimating lenses, the ion beam is tuned with a variable aperture, generating a beam current which is typically in a range from $1 p A$ to $1 n A$, leading to the possibility of performing very fine high resolution imaging or beam milling. When the focused gallium ion beam is raster scanned 


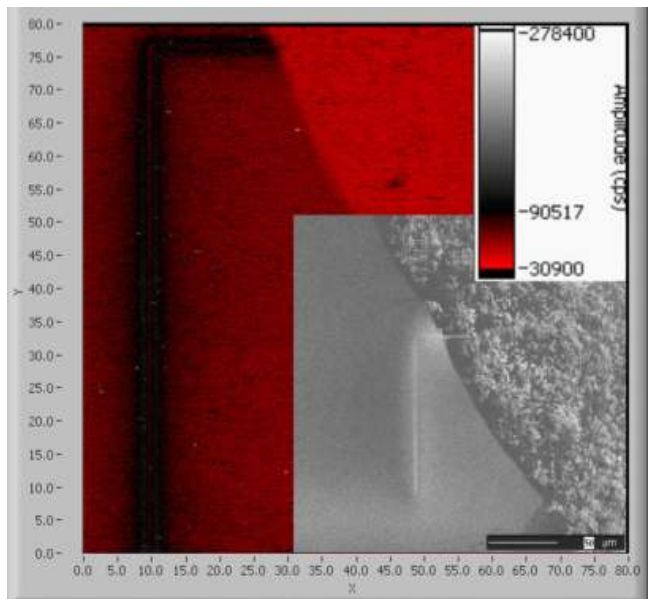

Figure 6. Confocal microscope image (pump 532nm filter $637 \pm 67 \mathrm{~nm}$ ) of the silver coated zone with a line etched on and next to it. (inset) secondary electron image.

over a substrate it causes electron emission which is collected on a biased high gain detector, called, a microchannel plate $(M C P)$. The detector bias is a positive or a negative voltage, respectively, for collecting secondary electrons or secondary ions. Detection can then be used to build up an image of the sample as the beam is scanned across it. The imaging taken in this way has resolution limited only by the focal spot size $(\sim 10 \mathrm{~nm})$. Moreover if the current of the beam is high, another effect also occurs; physical sputtering or milling of the sample material. By scanning the beam over the substrate, arbitrary shapes can be etched. The FIB offers the ability to perform nanopatterning, allowing design and prototyping of new micro or nanostructures. The ion beam itself could also be used to perform spatially confined Ga-doping.

\section{Fabrication of structures near $\mathrm{N}-\mathrm{V}$ centers, preliminary studies}

In [1] we have introduced the new procedure we used to create structures on demand coupled to $\mathrm{NV}^{-}$centres, this new technique but before relies on some promising preliminary results we are going to show here. These results indicate that etching with $F I B$ next to the $\mathrm{NV}^{-}$centres $(\sim 1 \mu \mathrm{m})$ does not affect their emission. In order to be sure that the FIB has not damaged the implanted $\mathrm{NV}^{-}$centres like for example in [21], we first cover the surface of the diamond with silver in the zone where the $\mathrm{NV}^{-}$centres were located. The silver cover zone acted as a shield for the gallium implantation and allowed us to check the difference in light emission from the $\mathrm{NV}^{-}$centres exposed to the Ion beam. Then we etched, with FIB, a deep long line next to the silver cover zone as shown in Fig. 6. After that we removed the silver coating, and analysed the possible damage to the $\mathrm{NV}^{-}$array. Fig. 7 shows the behaviour of count rate from single $\mathrm{NV}^{-}$centres taken at two different points, one in the region exposed to gallium and the other in the region covered by the silver after cleaning. Once we were assured that there is no damage to the array, we etched another line closer to the $\mathrm{NV}^{-}$array which was used as a reference in future procedures, and we repeated the procedure, confirming again that the FIB etching does not affect the $\mathrm{NV}^{-}$centre emission 


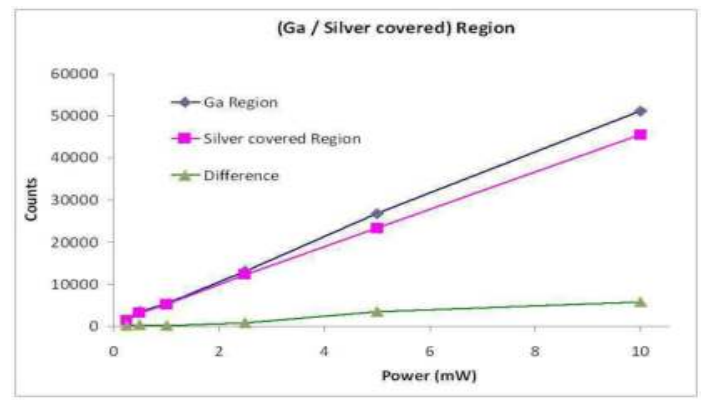

Figure 7. Count rate as function of laser power, measured after cleaning (section 10) the surface

because there were no changes in the count rates from the $\mathrm{NV}^{-}$. This procedures proves that etching next to the $\mathrm{NV}^{-}$centres (within $5 \mu \mathrm{m}$ ) does not create any damage to them.

\subsection{Platinum deposition}

Another important feature of the FIB machine is represented by the possibility of performing metal deposition onto the sample. The way it works is similar to chemical vapour deposition $(C V D)$ and the occurring reactions are comparable to, for example, laser induced $C V D$. The main difference is the better resolution but lower deposition rate of $F I B$. The metals that can be deposited on commercially available machines are platinum $(P t)$ and tungsten $(W)$. We mainly used platinum deposition in our etching sessions. By coating in Platinum we produce a conducting surface on the diamond which decreases the build up of charge due to deposition of $\mathrm{Ga}^{+}$. This then reduces the beam shift due to accumulation of the gallium ion charge during the milling process. The deposition process works as follows: a platinum-bearing organometallic precursor gas is sprayed on the surface by a fine needle (nozzle), where it adsorbs. In a second step, the ion beam decomposes the adsorbed precursor gases. Then the volatile reaction products desorb from the surface and are removed through the vacuum system leaving mostly platinum and small amount of organic solid at the surface. Auger electron observations have shown that this Platinum $(27 \%)$ is highly contaminated with Carbon (65\%) originated during the metal organic precursor decomposition and Gallium (8\%) coming form the ion beam. The deposition result is illustrated in Fig. 8 where we can see the platinum coated zone and the mouth of the injector needle. We used the platinum deposition in order to perform precise etching of desired pattern as we will see in the next section.

\section{Fabricating photonic crystals using focus ion beam etching}

Having simulated photonic crystal structure cavities we began fabrication via focused ion beam etching $(F I B)[22,23]$ our aim being to create a suspended membrane with the "Noda" cavity described previously. Other groups have performed fabrication with FIB in diamond of different kind of photonic crystal structures, some group performed also a measure of a $Q$ factor of $Q=535$ [24]. Here we try different approach in order to fabricate the "Noda" Cavity. In the first fabrication step, the diamond crystal is undercut by turning side-on and etching to obtain a $200 \mathrm{~nm}$ thick slab attached to the bulk (a suspended slab). In this stage 


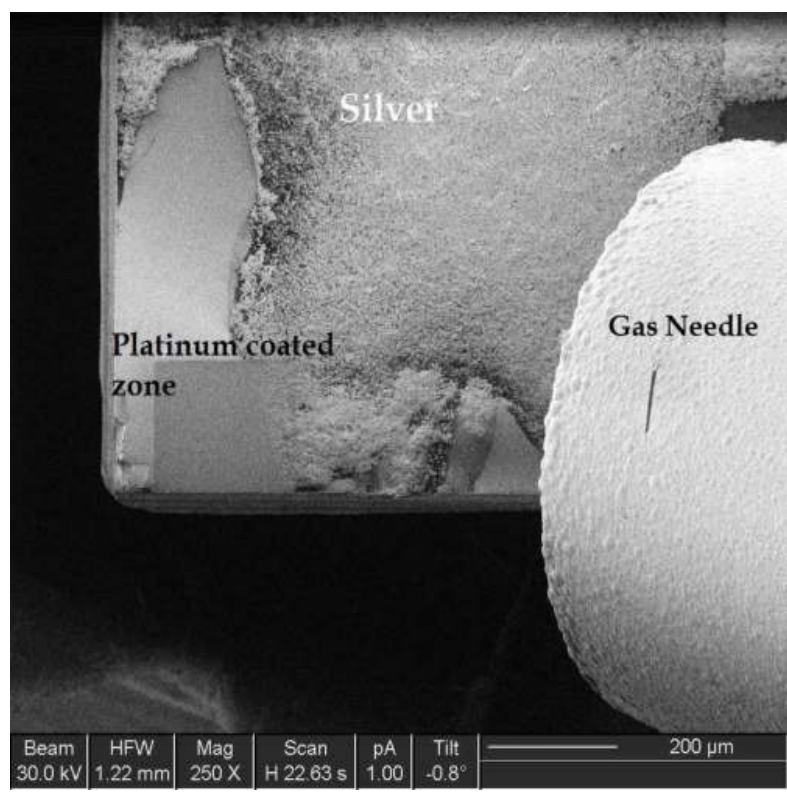

Figure 8. Secondary electron image of the sample with a square of Platinum coated zone made with gas deposition technique, the gas needle is also visible on the right hand of the picture.

the use of platinum deposition, as described before, is crucial in order to obtain precise structures. We needed to etch the membrane first, because if we had made the photonic crystal structure first, at the stage in which we etch the membrane some sputtering could have filled the holes. In order to etch the membrane we mounted the sample on a stage, and then tilted it to $90^{\circ}$. After we covered the $\mathrm{NV}^{-}$centre array zone with silver, in order to protect the implanted $\mathrm{NV}^{-}$swe etched a thin membrane of $200 \mathrm{~nm}$ according to the results of the simulation shown previously. In Fig.9(a) we can see a top view of the membrane and in 9 (b) we can see an image of the same membrane tilted by $45^{\circ}$. After we made the membrane we repositioned the sample horizontally and finally we etched the hexagonal air hole array with cavity formed from three filled holes. Fig. 10 shows two views, tilted $45^{\circ}$ at different magnifications, of the resulting structure. Both were secondary electron images taken with $F I B$ after the etching. In Fig. 10(a), we can see the photonic crystal cavity etched in the membrane. This is more evident in Fig. 10(b) where we have a scan over a larger area which shows the size of the cavities compared to the suspended membrane. In the top view, shown in Fig. 11(a), we can observe the cavity and notice some imperfections in it due to the FIB technique which creates deposits of etched material during the scanning. In Fig. 11(b) we can see an image taken with a confocal microscope, in which blue colour means low intensity and red colour means high intensity. Fig. 11(b) is remarkable because we can clearly see reduced fluorescence in the unetched zone, forming the cavity. Because there is no (or less) etch damage in these regions. This is an encouraging because it means that if there were an $\mathrm{NV}^{-}$centre in the cavity we might be able to see it. We performed some measurement of the spectrum of the light emitted from the cavity region. Unfortunately we were not able to see any enhancement of the signal as we might expect from a cavity resonance, but just a broad emission as shown in Fig. 12. At this stage we decided to take a step back and to 

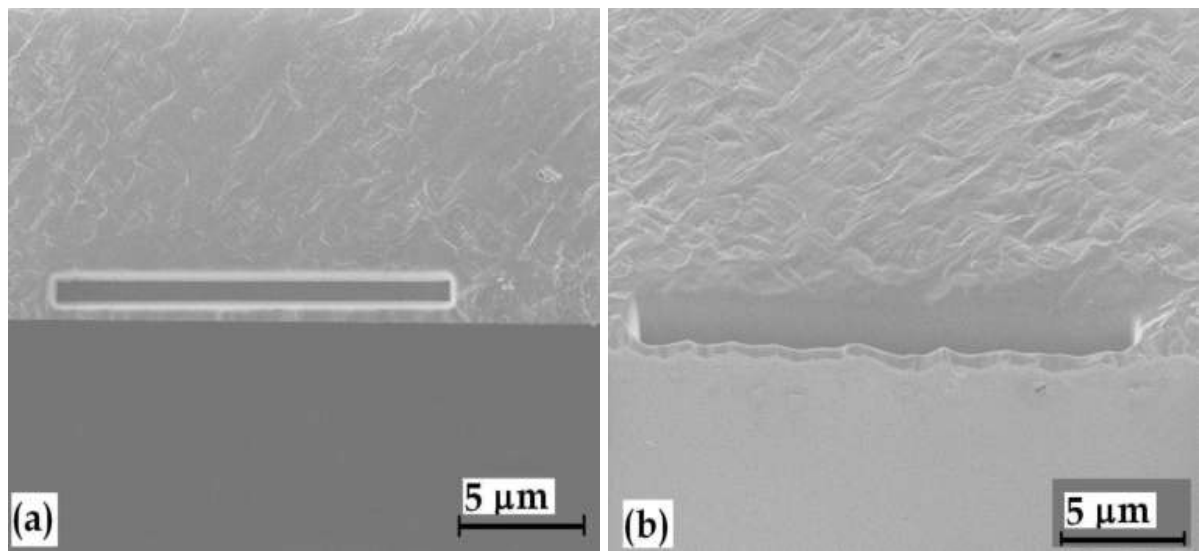

Figure 9. secondary electron image of a etched membrane in the diamond sample. a) a top view of the etched membrane b) $45^{\circ}$ Tilted view of the etched membrane

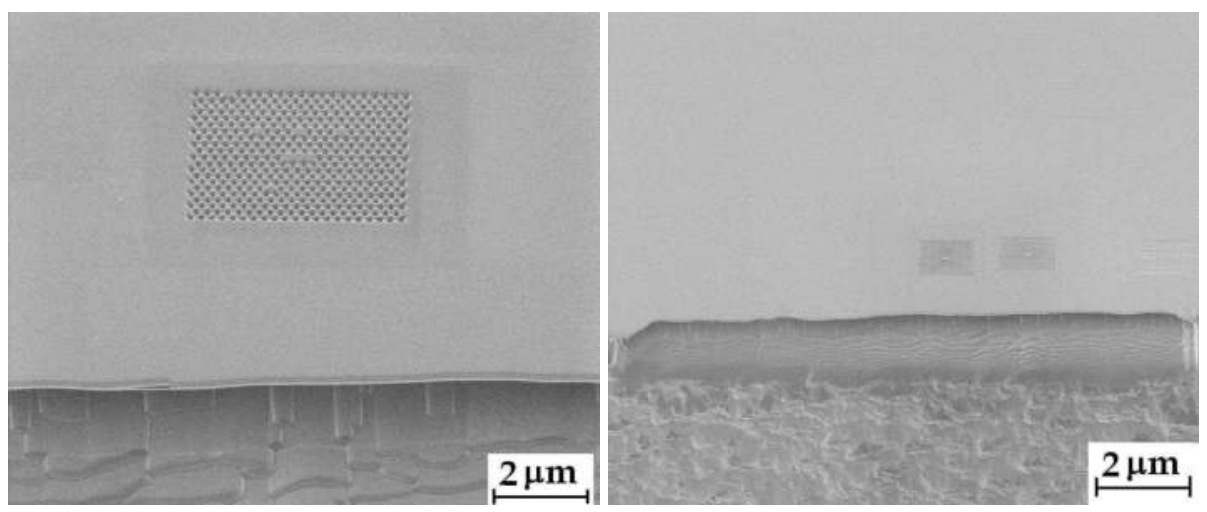

Figure 10. Secondary electron image of a etched photonic crystal structure in the diamond sample: a) Tilted view of the $L 3$ cavity taken with $F I B$ at different tilt and magnitude. b) larger image of the membrane and the cavities.

perform a preliminary study about the real possibility of coupling a single $\mathrm{NV}^{-}$centre to a larger structure etched in the diamond with FIB. This motivated our studies of the solid immersion lens as will be discussed in the next section.

\section{Fabricating solid immersion lenses using focused ion beam etching}

In this chapter we have studied how to fabricate the photonic crystal structure in order to create a cavity to enhance the coupling of $\mathrm{NV}^{-}$centres in diamond. As already discussed, $\mathrm{NV}^{-}$photon collection efficiency is severely reduced by losses due to the high refractive index of diamond. This is a problem regardless of the application, or the particular defect centre of interest. A possible solution we presented [25], is represented by the fabrication of hemispherical integrated solid immersion lenses (SILS) etched directly into the diamond surface. In order to avoid any scattering and absorption of the light emitted at high angles we need to have a SIL surrounded just by air. Hence we etch a SIL surrounded by a ring trench. 

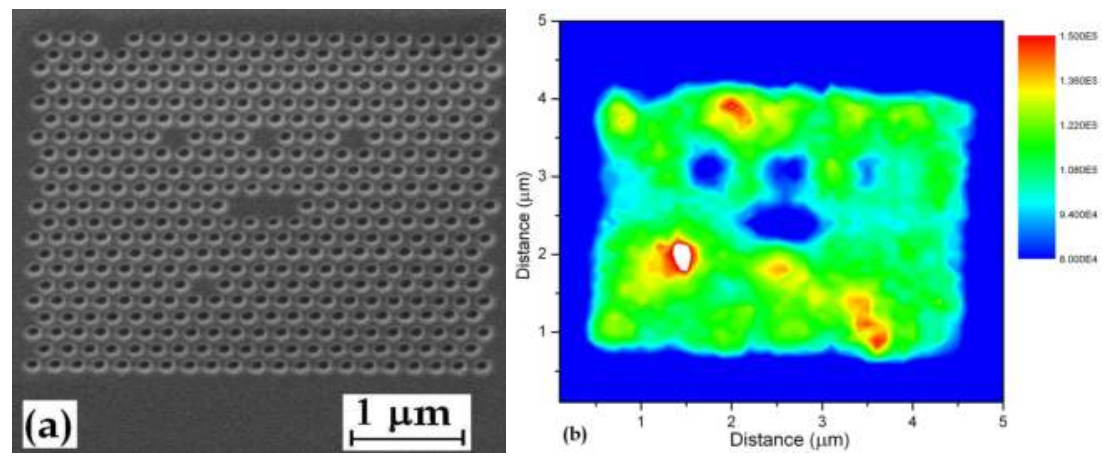

Figure 11. Photonic crystal structure in the diamond sample: a) Secondary electron emission image of the top view of the $L 3$ cavity (inset:zoom of the cavity). b) Fluorescence image taken with the confocal microscope (colour red: high intensity, colour blue:low intensity).

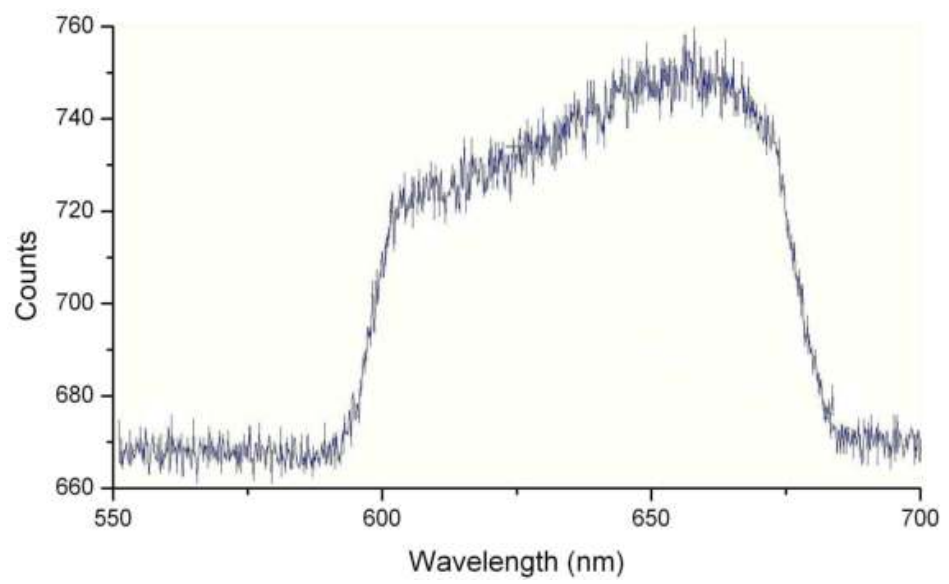

Figure 12. Image of the emission spectrum taken at the centre of the photonic crystal cavity, taken with a pass-band filter(semrock FF01 - 675/67 - 25) tilted a little bit in order to avoid raman scattering from the diamond. No narrow peaks are seen as would be expected from cavity resonance effects.

In order to do this, we have split the whole procedure into two different steps, etching a ring trench the depth of the SIL and then etching the SIL inside the ring. We etched a ring of a depth of $4 \mu \mathrm{m}$ with a current of $6.6 n \mathrm{~A}$. This part of the procedure is a routine etch with $F I B$. The challenge is represented by etching a hemispherical SIL in the centre of the ring. In order to do that we milled a series of concentric rings of increasing depth and diameter at the current of $1 n A$. We etched the material in a way different from the usual one: instead of etching from the top to bottom of the structure we milled from the centre moving the ion beam in a spiral way (trepanning), and then we repeated this for many concentric rings. The spiral technique allowed us to avoid a possible shift due to the etching process, giving us a SIL placed in the ring etched previously. Fig. 13(a) shows images of the SILs taken using secondary electron emission in the FIB system. We can see a a SIL in the centre of the image, just below the square, and a SIL inside a ring in the lower right/hand corner of the image. Fig. 13 b shows a $45 \circ$ tilted view of a SIL. Recently [1] we have shown how to fabricate a SIL 

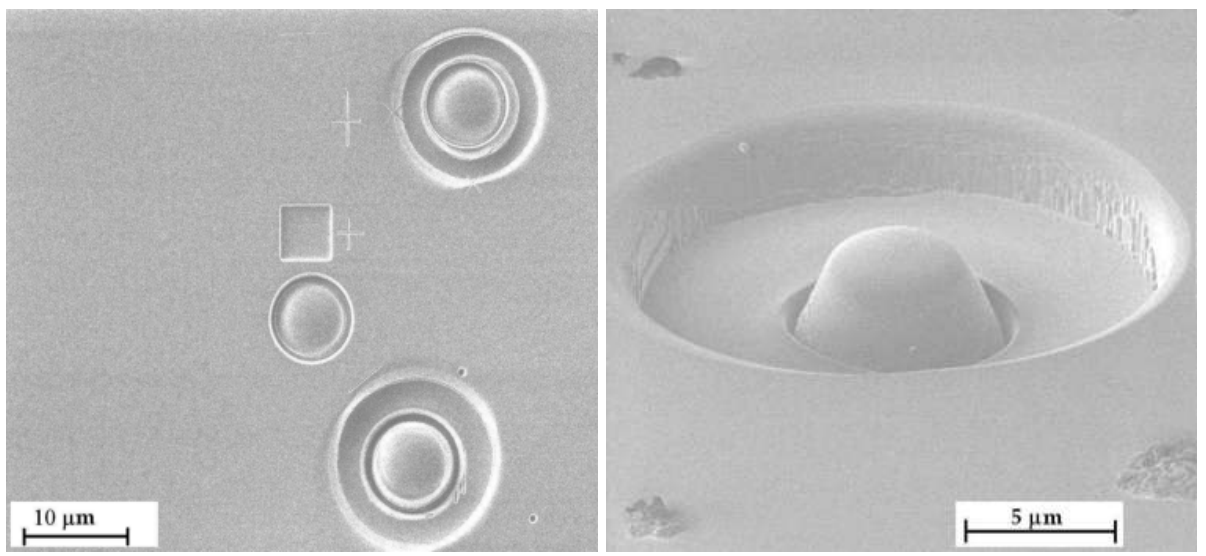

Figure 13. secondary electron emission images showing: a) top view of the SIL etched in the diamond b) $45^{\circ}$ tilted view of the SIL etched in the diamond
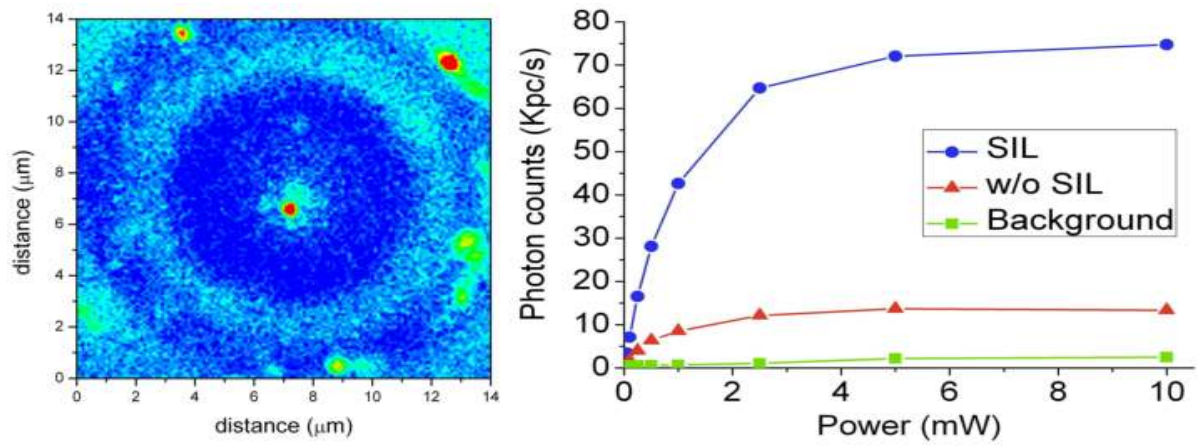

Figure 14. a)Photoluminescence image of the SIL with the enhanced emission from the $\mathrm{NV}^{-}$center taken at temperature $T=4.2 \mathrm{~K}, \mathrm{~b})$ Comparison of the different photoluminescence count rates as function of the laser intensity (image taken from [1]).

located over a previously identified $\mathrm{NV}^{-}$centre, without entering in detail, we just report the increasing of the collection of the light of $8 \times$, as shown in Fig.14b, from a Single $N^{-}$centre coupled to the SIL fabricated on demand on it as shown in Fig.14a

\subsection{Chemical cleaning of the diamond}

After each etching procedure it was essential to thoroughly clean the diamond surface. This dramatically reduced the background fluorescence intensity by removing all sputter deposits of gallium, platinum and other organic contaminants. We firstly rinsed the sample in a hydro-sonic bath three different times, each with a different component: the first time with acetone in order to remove the main dust, the second time with isopropanol to remove the acetone and finally with distilled water in order to remove the isopropanol. After we cleaned the most visible impurities, we put the diamond in a bath of $100 \mathrm{ml}$ of Sulphuric Acid $\left(\mathrm{H}_{2} \mathrm{SO}_{4}\right)$ and then heated it till it reached the temperature of $200^{\circ} \mathrm{C}$. Once the right temperature was 
achieved we added $5 \mathrm{mg}$ of potassium nitrate $\left(\mathrm{KNO}_{3}\right)$ leaving it for 20 minutes. The resulting reaction, is shown in Eq. 2. It is very aggressive and fast and creates a mixture of sulphuric acid and nitric acid at high temperature which removes all remaining contaminants leaving a very clean sample.

$$
\mathrm{H}_{2} \mathrm{SO}_{4}+\mathrm{NO}_{3}^{-} \rightarrow \mathrm{HSO}_{4}^{-}+\mathrm{HNO}_{3}
$$

\section{Conclusion}

In this work we discussed about the feasibility of $\mathrm{NV}^{-}$centers as single photon emitters and how to use its spin as qubit for quantum computing applications, remarking the many advantages that the use of $\mathrm{NV}^{-}$center in diamond would produce. One of the key challenges in order to perform a real implementation of a quantum computer concerns the possibility of handling the qubit. The spin of the ground state of the $\mathrm{NV}^{-}$center shows some characteristics we have described earlier in this work, which looks very promising for this purposes. One of the crucial step in order to perform spin readout and non demolition measurement of the spin of the $\mathrm{NV}^{-}$center is represented by increasing the coupling between the light and the solid state system. We showed a way to increase the coupling between the $\mathrm{NV}^{-}$and the light by placing the $\mathrm{NV}^{-}$center in a photonic crystal cavity. We characterized the photonic crystal cavity tuning it to be resonant with the $\mathrm{NV}^{-}$center emission, having had encouraging results in the simulation and fabrication of the cavity, in that we reached a reasonable high value of quality factor and small modal volume. Another very important aspect in order to build a quantum computer is represented by the possibility of handling a single photon source. In order to use $\mathrm{NV}^{-}$as single photon emitter, one of the challenges is represented by the light collection. We discussed in this work a solution we developed in order to increase the light collection from the $\mathrm{NV}^{-}$center by etching a solid immersion lens around it, proposing a technique ([1]) in order to locate $\mathrm{NV}^{-}$centers with accuracy of $10 \mathrm{~nm}$, and fabricate structure around them. In future works we will use the technique we have recently developed, in order to create a photonic crystal around a single $\mathrm{NV}^{-}$center. Another important path we want to follow consist in exploiting another useful color center in the diamond, the chromium center. This center acts as a single photon emitter as well but with a narrower spectrum. It has a resonant wavelength of $755 \mathrm{~nm}$ which is in the wavelength range for the Si photon counting detector, allowing us to detect them with high efficiency. We are very interested in using the technique we have developed in order to etch photonic crystal around single chromium center and coupling with it, this would allow us to increase the light collection from the chromium center permitting it to be used as an ultra bright single photon emitter. As it emits at $755 \mathrm{~nm}$ it is compatible with integrated photonic circuits being developed in our group by [26]. Similarly the nickel-nitrogen complex (NE8) center in diamonds, studied by [27, 28], has narrow emission bandwidth of $1.2 \mathrm{~nm}$ at room temperature with emission wavelength around $800 \mathrm{~nm}$, again suitable for Si detectors and quantum photonic circuits. In addition, in this spectral region little background light from the diamond bulk material is detected, which made it an interesting possible candidate for single photon source. Once we are able to locate an NE8 (or other suitable narrowband) center we will extend the registration procedure developed to allow fabrication of photonic crystal structure around individual defects, ending in the measurement of Q-factors and Purcell enhanced emission. In order to handle and guide the light emitted from the source a detailed study of parameters of a photonic crystal waveguide in the diamond will be required as demonstarted by [29]. We 
will simulate the behavior of the electromagnetic field inside the cavity and how it will couple with the waveguide. A good response will lead us to fabricate and then measure the effective coupling. We will also explore different etching techniques such as Reactive-Ion Etching (RIE) which will allow us to create membranes and very thin structures in diamond with a high precision. This will be useful in order to create different structures around registered $\mathrm{NV}^{-}$centers, for instance with photonic crystal nanobeam cavities studied by [30]. This kind of structures as the remarkable advantage to be very easy to fabricate offering a huge quality factor and a very small modal volume.

\section{Author details}

Luca Marseglia

Institut für Quantenoptik, Universität Ulm, Ulm, Germany

\section{References}

[1] L Marseglia, J P Hadden, A C Stanley-Clarke, J P Harrison, B Patton, Y-L D Ho, B Naydenov, F Jelezko, J Meijer, P R Dolan, J M Smith, J G Rarity, and J L O’Brien. Nano-fabricated solid immersion lenses registered to single emitters in diamond. Appl. Phys. Lett., 98:133107, 2011.

[2] T Gabel, M Dohman, I Popa, C Wittmann, P Neumann, F Jelezko, J R Rabeau, N Stavrias, A D Greentree, S Prawer, J Meijer, J Twamley, P R Hemmer, and J Wrachtrup. Room-temperature coherent coupling of single spins in diamond. Nature Physics, 2:408 $-413,2006$.

[3] C Kurtsiefer, S Mayer, P Zarda, and H Weinfurter. Stable solid-state source of single photons. Phys. Rev. Lett., 85:290, 2000.

[4] R Hanson, F M Mendoza, R J Epstein, and D D Awschalom. Polarization and readout of coupled single spins in diamond. Phys. Rev. Lett., 97:087601, 2006.

[5] E van Oortt, N B Manson, and M Glasbeekt. Optically detected spin coherence of the diamond n-v centre in its triplet ground state. J. Phys. C: Solid State Phys., 21:4385-4391, 1988.

[6] A Gruber, A Dräbenstedt, C Tietz, L Fleury, J Wrachtrup, and C von Borczyskowski. Scanning confocal optical microscopy and magnetic resonance on single defect centers. Science, 276, 1997.

[7] A Young, C Y Hu, L Marseglia, J P Harrison, J L O'Brien, and J G Rarity. Cavity enhanced spin measurement of the ground state spin of an nv center in diamond. New Journal of Physics, 11:013007, 2009.

[8] C F Wang, R Hanson, D D Awschalom, E L Hu, T Feygelson, J Yang, and J E Butler. Fabrication and charcterization of two-dimensional photonic crystal microcavities in nanocrystalline diamond. Appl.Phys.Lett., 91:201112, 2007. 
[9] F Jelezko, T Gaebel, I Popa, A Gruber, and J Wrachtrup. Observation of coherent oscillation of a single nuclear spin and realization of a two-qubit conditional quantum gate. Phys. Rev. Lett., 93:7, 2004.

[10] F Jelezko, T Gaebel, I Popa, A Gruber, and J Wrachtrup. Observation of coherent oscillations in a single electron spin. Phys. Rev. Lett., 92:7, 2004.

[11] A Gali, M Fyta, and E Kaxiras. Ab initio supercell calculations on nitrogen-vacancy center in diamond: Electronic structure and hyperfine tensors. Physical Review B, 77:155206, 2008.

[12] N B Manson, J P Harrison, and M J Sellars. Nitrogen-vacancy center in diamond: Model of the electronic structure and associated dynamics. Phys. Rev. B, 74:104303, 2006.

[13] P Tamarat, N B Manson, J P Harrison, R L McMurtrie, A Nizovtsev, C Santori, R G Beausoleil, P Neumann, T Gaebel, F Jelezko, P Hemmer, and J Wrachtrup. Spin-flip and spin-conserving optical transitions of the nitrogen-vacancy centre in diamond. New Journal of Physics, 10:045004, 2008.

[14] F Jelezko and J Wrachtrup. Read-out of single spins by optical spectroscopy. J. Phys.: Condens. Matter, 16:104, 2004.

[15] C H Su, A D Greentree, and L C L Hollenberg. Towards a picosecond transform-limited nitrogen-vacancy based single photon source. Optics Express, 16:6240, 2008.

[16] P Neumann, N Mizuochi, F Rempp, P Hemmer, H Watanabe, S Yamasaki, V Jacques, T Gaebel, F Jelezko, and J Wrachtrup. Multipartite entanglement among single spins in diamond. Science, 320:1326, 2008.

[17] L Marseglia. Photonic crystals - innovative systems, lasers and waveguides. InTech, ISBN 978-953-51-0416-2, 2012.

[18] A A Tseng. Recent developments in micromilling using focused ion beam technology. J. Micromech. Microeng., 14:15-34, 2004.

[19] J Melngailis. Critical review: focused ion beam technology and applications. J. Vac. Sci. Technol. B, 5:469, 1987.

[20] S Reyntjens and R Puers. A review of focused ion beam applications in microsystem technology. J. Micromech. Microeng., 11:287?300, 2001.

[21] S Rubanov, P Munroe, S Prawer, and D Jamieson. Surface damage in silicon after 30kev ga fib fabrication. Microscopy and Microanalysis, 9:884, 2003.

[22] D P Adams, M J Vasile, T M Mayer, and V C Hodges. Focused ion beam milling of diamond: Effects of h2o on yield, surface morphology and microstructure. J. Vac. Sci. Technol. B, 21, 2003. 
[23] J Taniguchi, $\mathrm{N}$ Ohno, $\mathrm{S}$ Takeda, I Miyamoto, and $\mathrm{M}$ Komuro. it focused-ion-beam-assisted etching of diamond in xef2. J. Vac. Sci. Technol. B, 16, 1998.

[24] C F Wang, Y S Choi, J C Lee, E L Hu, J Yang, and J E Butler. Observation of whispering gallery modes in nanocrystalline diamond microdisks. Appl. Phys. Lett., 90:081110, 2007.

[25] J P Hadden, J P Harrison, A C Stanley-Clarke, L Marseglia, Y-L D Ho, B R Patton, J L O'Brien, and J G Rarity. Strongly enhanced photon collection from diamond defect centres under micro-fabricated integrated solid immersion lenses. Appl. Phys. Lett., 67:241901, 2010.

[26] A Politi, M J Cryan, J G Rarity, S Yu, and J L O'Brien. Silica-on-silicon waveguide quantum circuits. Science, 320:646-649, 2008.

[27] J R Rabeau, Y L Chin, S Prawer, F Jelezko, T Gaebel, , and J Wrachtrup. Fabrication of single nickel-nitrogen defects in diamond by chemical vapor deposition. Appl. Phys. Lett., 86:131926, 2005.

[28] T Gaebel, I Popa, A Gruber, M Domhan, F Jelezko, and J Wrachtrup. Stable single-photon source in the near infrared. New Journal of Physics, 6:98, 2004.

[29] B-S Song, S Noda, and T Asano. Photonic devices based on in-plane hetero photonic crystals. Science, 300:1537, 2007.

[30] P B Deotare, M W McCutcheon, I W Frank, M Khan, and M Loncar. Coupled photonic crystal nanobeam cavities. Appl. Phys. Lett., 95:031102, 2009. 
